Check for updates

Cite this: Phys. Chem. Chem. Phys., 2020, 22, 12267

Received 10th April 2020, Accepted 7th May 2020

DOI: $10.1039 / \mathrm{d} 0 \mathrm{cp01956a}$

rsc.li/pccp

\title{
Molecular environment and reactivity in gels and colloidal solutions under identical conditions $\dagger$
}

\author{
Philip Groves, ${ }^{a}$ Jin Huang, ${ }^{b}$ Andreas Heise, (D) ${ }^{c}$ Jennifer Marsh ${ }^{d}$ and \\ Victor Chechik iD *a
}

\begin{abstract}
A PEG-Tyr block copolymer forms a kinetically stable colloidal solution in water at room temperature which undergoes an irreversible conversion to a gel phase upon heating. A micellar solution and a gel can therefore be studied under identical experimental conditions. This made it possible to compare physical properties and chemical reactivity of micelles and gels in identical chemical environments and under identical conditions. EPR spectra of the spin-labelled copolymer showed that tyrosine mobility in gels was slightly reduced compared to micelles. Chemical reactivity was studied using photochemical degradation of tyrosine and tyrosine dimerization, in the absence and in the presence of an Fe(III) salt. The reactivity trends were explained by reduced tyrosine mobility in the gel environment. The largest reactivity difference in gels and micelles was observed for bimolecular dityrosine formation which was also attributed to the reduction in molecular mobility.
\end{abstract}

\section{Introduction}

Supramolecular assembly has a profound effect on molecular environment. Solvation and dynamics are key factors determining many chemical and physical properties of supramolecular structures such as host-guest complexes, micelles, or gels. ${ }^{1}$ Chemical properties of functional groups can also be significantly affected by intermolecular interactions in supramolecular assemblies. The most remarkable examples of unusual chemical reactivity are in host-guest complexes, ${ }^{2}$ but the reactivity can also be altered in less well defined systems such as micelles or gels. ${ }^{3}$ Obtaining quantitative understanding of the factors determining reactivity in such supramolecular assemblies is challenging, not least because reference points do not exist under identical conditions.

Importantly, supramolecular assembly of large (e.g., polymeric) building blocks is often almost irreversible at room temperature. ${ }^{4}$ These assemblies may become kinetically trapped and do not spontaneously convert into a more thermodynamically stable form. Such systems present a unique opportunity to examine the

\footnotetext{
${ }^{a}$ Department of Chemistry, University of York, Heslington, York YO10 5DD, UK. E-mail: victor.chechik@york.ac.uk

${ }^{b}$ School of Chemical Sciences, Dublin City University, Dublin 9, Ireland

${ }^{c}$ Department of Chemistry, Royal College of Surgeons in Ireland, 123 St. Stephens Green, Dublin 2, Ireland

${ }^{d}$ The Procter \& Gamble Company, Mason Business Center, 8700 Mason-Montgomery Road, Mason, 45040, USA

$\dagger$ Electronic supplementary information (ESI) available: Additional experimental data, EPR spectra and simulation details. See DOI: 10.1039/d0cp01956a
}

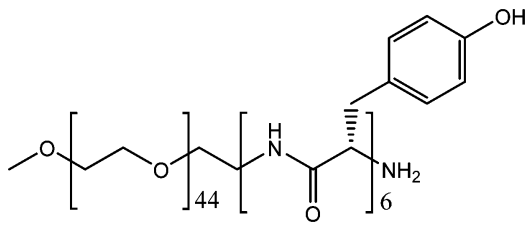

Fig. 1 PEG-L-Tyr block copolymer system PEG2000-Tyr 6 .

properties of chemically-identical supramolecular systems which are kinetically trapped in different morphologies.

PEG-Tyr block copolymer (Fig. 1) is an example of such a supramolecular system. ${ }^{5}$ The hydrophilicity of the PEG block and hydrophobicity of the Tyr block make the copolymer selfassemble into kinetically-trapped, stable colloidal structures upon dissolution in water. Upon heating, the solutions spontaneously convert into more thermodynamically-stable gels. Hence, the colloidal solutions and the gels of this copolymer can be examined under identical experimental conditions. Here, we report the results of our study on the comparative reactivity and molecular dynamics (assessed by EPR spectroscopy) of colloidal solutions and gels of PEG-Tyr block copolymers.

\section{Results and discussion}

Characterisation of PEG-Tyr self-assembled aggregates

Aqueous solutions of the PEG2000-Tyr 6 copolymer was analysed by dynamic light scattering (Fig. 2). The polymer was found to form aggregates at room temperature with $c a$. 50-80 nm hydrodynamic diameter. As the volume of the long PEG chain relative 


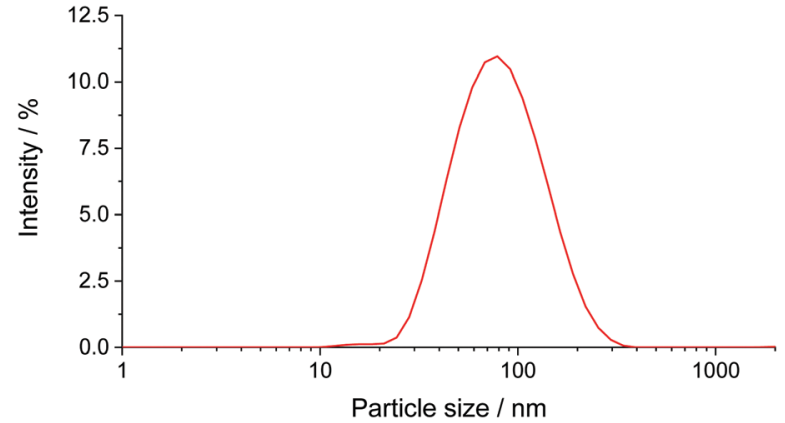

Fig. 2 DLS size distribution plot (by intensity) for PEG2000-Tyr 6 polymer sample in water. The plot is an average of 3 measurements.

to the overall surfactant volume is large, the colloidal structures are likely to be spherical micelles as suggested by TEM studies of PEG-Tyr copolymers. ${ }^{6}$

The critical micelle concentrations (cmc) of PEG2000-Tyr 6 was estimated from DLS measurements by plotting particle count rate $v s$. polymer concentration as $0.6 \times 10^{-5} \mathrm{~mol} \mathrm{dm}^{-3}$, which is equivalent to $0.017 \mathrm{mg} \mathrm{mL}^{-1}$ (ESI, $\dagger$ Fig. S1). Although this measurement only provides a crude estimate of $\mathrm{cmc}$, it is $c a$. 3 orders of magnitude lower than the concentrations used in all experiments reported here; hence it was safe to assume that polymer chains in our experiments are predominantly found in the micelles. Colloidal solutions of polymers were indefinitely stable at room temperature but formed gels upon heating to $60{ }^{\circ} \mathrm{C}$. The gels remained stable after cooling to room temperature.

Gel structures formed upon heating of PEG2000-Tyr 6 were previously characterised by Heise et $a .^{5}$ The cryo-TEM images showed a continuous, interconnected network of fibres with Tyr block adopting a typical $\beta$-sheet conformation as evidenced by FT-IR and CD spectroscopy.

\section{Spin labelling of PEG-Tyr copolymers}

In order to characterise the molecular dynamics of supramolecular assemblies of PEG-Tyr copolymers, they were spin-labelled with TEMPO units following a modified Mannich-type reaction protocol reported by Belle et al. (Scheme 1). ${ }^{7}$

Spin labelling of tyrosine residues within the micelle structure was not successful when the reaction was attempted in aqueous phosphate buffer solution. It was postulated that the micelle structures hinder access to the aromatic ring in tyrosine. The reaction was therefore repeated in methanol where copolymer

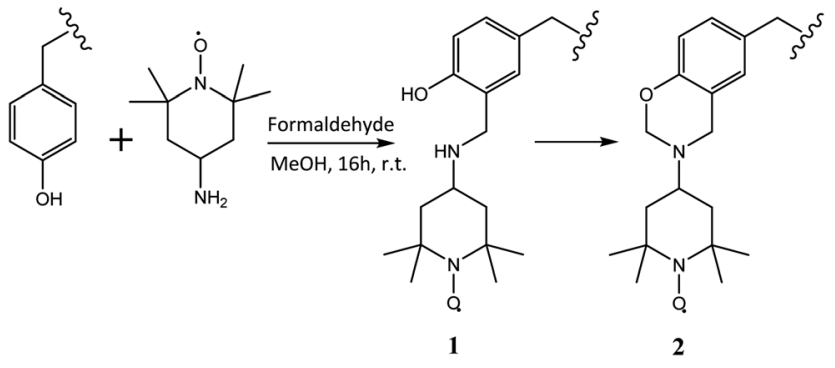

Scheme 1 Spin labelling of PEG-Tyr block copolymer tyrosine side chains with 4-amino-TEMPO. aggregation was at least partially suppressed (ESI, $\dagger$ Fig. S7). Aqueous solutions of spin-labelled polymer were purified from excess spin label by dialysis against water, as the colloidal assemblies were retained by the membrane. Purification by dialysis was monitored by EPR. No loss in polymer was observed during dialysis as confirmed by tyrosine fluorescence at $310 \mathrm{~nm}$.

The yield of the labelling reaction was estimated from the EPR signal intensity of the colloidal solution. The results indicate that $0.3 \%$ of available tyrosine residues were successfully labelled. Hence, a $5 \mathrm{mg} \mathrm{mL}{ }^{-1}$ solution of the polymer has the average nitroxide concentration of $c a .25 \mu \mathrm{M}$. This low yield is advantageous for an EPR study as interactions between adjacent labels can be ignored, and the concentration of labelled residues is still sufficiently high to give strong EPR signals.

\section{Characterisation of tyrosine microenvironment in micelles and gels}

Spin labelled PEG2000-Tyr 6 micelle and gel samples were analysed by X-band EPR spectroscopy. Spectra (Fig. 3) show overlapped contributions from several nitroxide environments. Rapidly tumbling radicals give rise to a characteristic three-line nitroxide spectrum, however both micelles and gels of the spin labelled polymer also exhibit nitroxides in significantly restricted environments, giving rise to broad features in the spectrum.

We postulate that the more restricted nitroxide environments observed in the spectra correspond to labelled tyrosine buried within a hydrophobic micelle core, or within a rigid gel fibre. There are notable qualitative differences between micelle and gel spectra, with the latter exhibiting clearer defined immobilised component peaks in low $(\approx 322.5 \mathrm{mT})$ and high $(\approx 329 \mathrm{mT})$ field regions. This indicates a slightly different tumbling environment in gel fibres compared to micelles.

The faster tumbling component likely represents spin labels at the surface of micelles/gel fibres; due to low cmc (vide infra), the concentration of dissolved unaggregated polymer is low and cannot give rise to this signal. The more flexible, acyclic Mannich adduct 1 (Scheme 1) which has been reported to incompletely convert to 2 in some cases ${ }^{8}$ could also contribute to this component.

The experimental X-band EPR data were simulated using EasySpin $^{9}$ to determine rotational diffusion parameters for the spin labelled micelles and gels. As the polarity of the spin label in the micelles/gels is unknown (and different from water), the principal values of $A$ and $g$ tensors of the spin-labelled systems could not be taken from the literature. They were instead

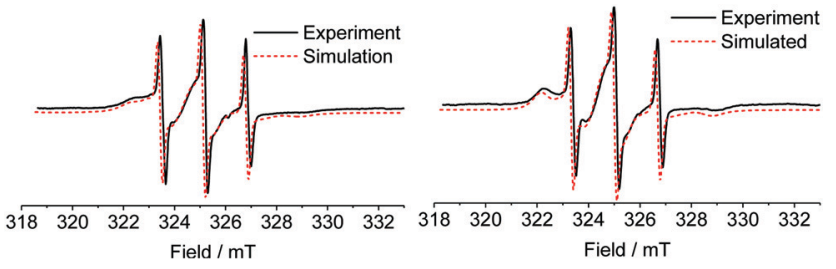

Fig. 3 X-band EPR spectra of spin labelled PEG2000-Tyr 6 micelles (left) and gel (right). Experimental data is shown in black solid line, fitted simulations in red dashed line. The simulations are offset for clarity. 
Table 1 Magnetic parameters of spin labelled PEG2000-Tyr 6 nitroxide radical calculated by fitting simulation to $Q$ band EPR spectrum of frozen solution

\begin{tabular}{llll}
\hline & $x$ & $y$ & $z$ \\
\hline$g$ & 2.00917 & 2.00579 & 2.00214 \\
$A / \mathrm{MHz}$ & 21.7 & 17.5 & 102.9
\end{tabular}

determined from the simulations of EPR spectra of frozen solution at Q-band (Table 1 and ESI, $\dagger$ Fig. S2a).

Attempts to simulate X-band EPR spectra of micelles and gels with a two-site model (with two different diffusion parameters, either isotropic or anisotropic) failed to give a satisfactory fit. This is not unexpected as there is clearly a complex distribution of labelling sites in the micelles and gels. A satisfactory fit was obtained with a simple three-site model which considers one fast tumbling component and two slow tumbling components. The tumbling of all components was assumed to be axiallysymmetric. The results of simulation are shown in Fig. 3 . The accuracy of our approach was tested by multifrequency analysis: a room-temperature $\mathrm{Q}$ band spectrum of a micellar sample was compared with a simulated spectrum with the same parameters, giving a reasonable fit (ESI, $\dagger$ Fig. S3).

Although the three-component simulation offers a reasonable fit of experimental data, it is an approximation of the distribution of different environments observed. The fitting algorithm compensates for this by broadening spectral linewidth. Therefore, accurate analysis of line shape is not possible. However, effective rotational diffusion rates and the relative contributions of different components could be qualitatively compared, as summarised in Table 2.

The tumbling rates in Table 2 are the averages of the perpendicular and parallel axial terms. Full data and linewidth for simulations is given in ESI, $\dagger$ Table S1a and c. Overall, tumbling is more restricted in gel fibres, as both the "fast" and "slow B" components possess lower tumbling rates $\mathrm{D}$ (Table 2) than those of the micelles. The relative contributions of the two "slow" components in the gel system highlights the slightly narrower distribution of immobilised nitroxide species compared to micelles, indicative of a more homogenous environment in gels. Overall, PEG-Tyr gels provide a more consistent tyrosine environment, which is slightly more restrictive than the micellar system.

As EPR studies of site-directed spin-labelled proteins reported in literature usually use different nitroxide spin labels with different flexibility, ${ }^{10}$ the scope for direct comparison of Tyr environment in model systems and protein structures is limited. However, labelled tyrosine near a flexible C-terminus in a small chloroplastic protein CP12 gives X-band EPR spectra similar to those

Table 2 Average rotational diffusion rates for individual components and their weightings

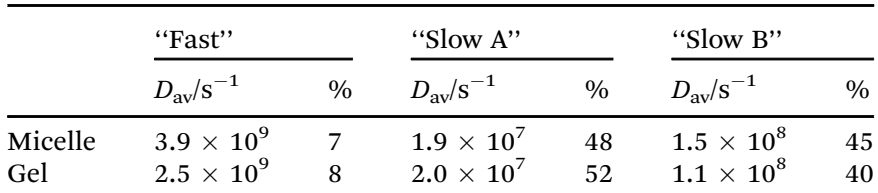

in Fig. $3^{8}$ and faster than the "slow" component in gels/micelles. This suggests that the environment of tyrosine within micellar and gel model systems is comparable to that in protein structures.

\section{Using spin labelled system to monitor transformation between micelle and gel}

The unique properties of the PEG-Tyr polymer system, coupled with the observable changes in spin label environment upon gelation, provide an opportunity to monitor the process of gel formation in situ. We followed gelation on three different scales: on a bulk scale using tube inversion test, on a supramolecular scale with DLS, and on a molecular scale with EPR spectroscopy. When a $10 \mathrm{mg} \mathrm{mL}{ }^{-1}$ colloidal solution of PEG2000-Tyr ${ }_{6}$ was rapidly heated to $60{ }^{\circ} \mathrm{C}$ and then kept at this temperature, a stable gel was formed after 8-10 minutes (tube inversion test). DLS measurements of samples rapidly heated and then kept at $60{ }^{\circ} \mathrm{C}$, on the other hand, indicate a rapid transformation of micelles into larger aggregate structures after only 30 seconds, with longer incubation at $60{ }^{\circ} \mathrm{C}$ leading to larger average particle size as gel fibres form (see ESI, $\dagger$ Fig. S4a and $b$ ). This suggests the rapid formation of loosely aggregated structures such as $\beta$-sheets (predicted by circular dichroism studies), significantly before bulk gelation occurs. ${ }^{5}$ DLS data become unreliable in the presence of very large particles, therefore only the initial phase of micelle breakdown could be accurately monitored.

Molecular changes during micelle-gel transformation of spin-labelled polymer solution were monitored by EPR. Following gelation in situ at $60{ }^{\circ} \mathrm{C}$ proved challenging as the EPR spectra at this temperature show rapid tumbling and gelation is not accompanied by an appreciable change in the spectral lineshape. Therefore, samples were rapidly heated to $60{ }^{\circ} \mathrm{C}$ and then kept at this temperature for $30-120$ second time intervals for a period of 20 minutes, with spectra recorded at $20{ }^{\circ} \mathrm{C}$ after rapid cooling. The relative contribution of immobilised spectral components was found to change significantly with heating time as micelle structures collapsed and gel fibres formed, as outlined in Fig. 4.

We believe that changes reported in Fig. 4 reflect evolution of molecular environment during micelle-gel transition. A potential artefact which would have been caused by thermal conversion of $\mathbf{1}$ to $\mathbf{2}$ under these conditions can be ruled out as (i) this conversion requires formaldehyde which is not present in the reaction mixture, and (ii) the changes to EPR spectra upon gelation are completely reversible (e.g., dissolution of gel in methanol
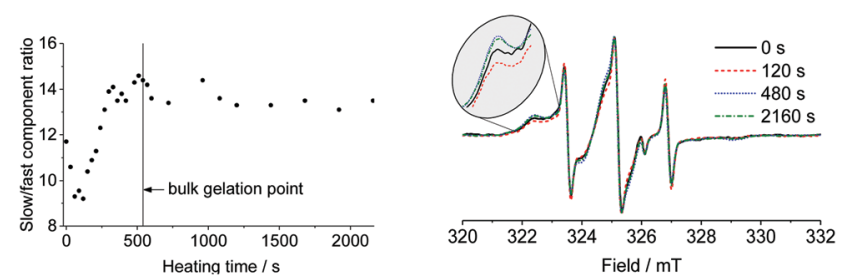

Fig. 4 Change in combined slow components contribution to EPR spectrum relative to fast component upon heating over time (left). Selected normalised EPR spectra (right). 
followed by evaporation and resuspension in water gives EPR spectra identical to those of original micelles, see ESI, $\dagger$ Fig. S5).

Interestingly, the initial heating stage leads to a rapid loss in immobilised nitroxide environment (22\% drop) over 120 seconds (Fig. 4) as micelle structures break down. This loose aggregate intermediate is probably more mobile than micelle or gel components, and could therefore correspond to a sheet-type structure, consistent with DLS data. There is still significant immobilised component, likely due to the formation of larger aggregate structures observed at longer timescales by DLS. Continued heating leads to a gradual increase in this immobilised component as loose aggregates form gel fibres up to a plateau after 10 minutes. This is in good agreement with macroscopic observations of gel formation. EPR results thus reveal a two-stage process of micelle-gel transformation: (i) breaking down of micelles, and (ii) re-assembly of loose aggregates into gel fibres.

\section{Photochemical degradation of gels and micelles}

In order to compare the reactivity of polymers in gels and micelles under identical reaction conditions, the reactions need to be triggered in both systems at a defined time. We argue that a photochemical reaction is best suited for such studies. As tyrosine undergoes a well-understood photodegradation which can be conveniently monitored by fluorescence, the gel and micelle samples were exposed to UV light (using high pressure UV lamp) for $24 \mathrm{~h}$. The characteristic tyrosine fluorescence was then analysed by fluorometry relative to a control sample kept in the dark. This provided information on relative reactivity of photoexcited tyrosine in micelles and gels. Dityrosine formation (a common tyrosine photooxidation product) ${ }^{11}$ was also monitored by fluorometry. This reported on bimolecular reactivity between two components of the supramolecular assemblies.

Gels and micelles have similar UV spectra. Although increased scattering in gels leads to somewhat higher absorbance, the absorbance at $276 \mathrm{~nm}$ (corresponding to tyrosine $\lambda_{\max }$ ) is very high and nearly identical in both systems (transmittance is $0.133 \%$ for micelles and $0.116 \%$ for gels, see ESI, $\dagger$ Fig. S6). Therefore, most of the light is absorbed by tyrosine rather than scattered, and the differences between micelles and gel absorbance are minimal.

Tyrosine degradation was slower in gel systems than micelles (Fig. 5, left). However, the difference is small. As the chemical environment and polymer concentration are identical in gel and micelle measurements, and the degradation reactions are kinetically limited by the absorption of light, the similar

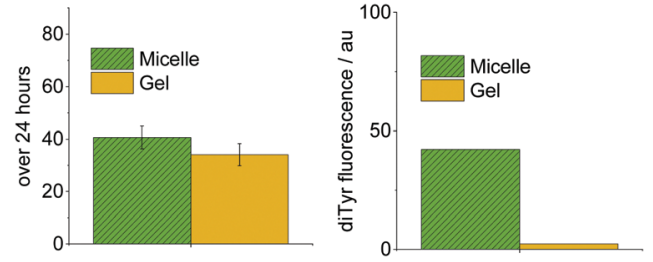

Fig. 5 Comparison of photochemical reactivity in $5 \mathrm{mg} \mathrm{mL}^{-1}$ micelle and gel systems for PEG2000-Tyr 6 . Measured by tyrosine degradation (left) and dityrosine formation (right).

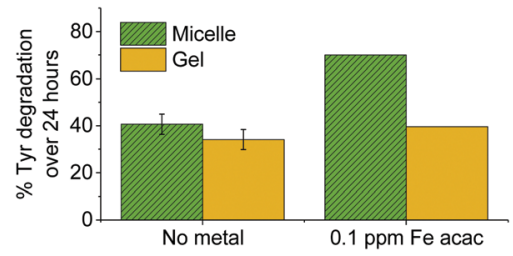

Fig. 6 Comparison of photochemical reactivity in PEG2000-Tyr 6 gel and micelle polymer systems in the presence and absence of $0.1 \mathrm{ppm} \mathrm{Fe}(\mathrm{III})$, measured by decrease in tyrosine fluorescence after 24 hours UV exposure.

photodegradation rates are not unexpected. The small difference must arise from the slightly more restricted physical environment of tyrosine in gel systems, which could affect processes after photoexcitation, e.g., reactions with oxygen and reactive oxygen species such as hydroxyl radicals, singlet oxygen and superoxide. ${ }^{12}$ These reactive species do not diffuse by more than a few nanometers in biological systems. ${ }^{13}$

The difference in reactivity is considerably more pronounced when dityrosine formation is monitored (Fig. 5, right). Gel systems show considerably lower formation of dimerised product. This is attributed to the slightly more restrictive physical environment in gels. As two tyrosyl radicals need to combine to form dityrosine, the dependence upon tyrosine mobility is much greater than for overall tyrosine degradation.

To test if gel systems limited diffusion of reactive oxygen species through the fibres, comparative photodegradation experiments were carried out on micelle and gel systems containing $0.1 \mathrm{ppm}$ tris(acetylacetonato)iron(III). This significantly increases the reactive oxygen species (ROS) flux through photo-Fenton processes. In addition, metal complexes could act as sensitisers for ROS production. ${ }^{14}$ The relatively hydrophobic metal complex likely incorporates into micelle cores and hydrophobic gel pockets. Direct interaction between metal centres and tyrosine is possible, however as the concentration of metal $\left(1.79 \mu \mathrm{mol} \mathrm{dm}{ }^{-3}\right)$ is very low compared to the polymer $\left(1.72 \mathrm{mmol} \mathrm{dm}^{-3}\right)$, this is likely to be diffusion limited.

The presence of the redox active metal significantly increases tyrosine degradation in micelle systems, as shown in Fig. 6, however gel systems show only a modest increase in activity. The lower tumbling rates of gels compared to micelles in our EPR studies (vide supra) suggested that gel fibres have lower mobility compared to micelles. Hence they restrict diffusion of reactive oxygen species generated at metal centres, limiting their effectiveness over larger distances. Diffusion of the metal complex is likely also restricted leading to further reduction in gel reactivity.

\section{Conclusions}

An amphiphilic PEG-Tyr copolymer forms two distinct physical environments (a micellar solution and a gel) which are kinetically stable under ambient conditions. This provided a unique opportunity to study the effect of physical and molecular environment upon chemical reactivity, in chemically identical systems. Using EPR spectroscopy of spin-labelled copolymers, 
we observed co-existence of a relatively mobile and a relatively immobile environments in both morphologies. Two stages were observed during the transformation of micelles to gels: breaking up of micelles into loose aggregates and their assembly into fibres. Overall, tyrosine units were slightly less mobile in a gel than in a micelle morphology, although the microenvironments are quite similar.

Photochemical degradation of tyrosine and formation of ditorysine was then studied in gels and micelles in the absence and in the presence of a relatively hydrophobic Fe(III) salt. Reactivity was fairly similar in both morphologies, with reactions in gels consistently slower than in micelles. This is consistent with the slightly lower tumbling rates (and hence lower mobility) of spin-labelled tyrosine units observed by EPR. The largest reactivity difference was observed for formation of dityrosine which requires two tyrosine units on polymer chains to come together: ditorysine formation in gels was much less pronounced than in micelles. This is also consistent with the lower tyrosine mobility in the gel environment.

Our study of micelle and gel reactivity in identical chemical environments showed relatively small differences, despite very different bulk appearance ("solid" gels vs. "liquid" colloidal solutions). The reactivity can be readily explained by the molecular mobility in the microenvironment around the reactive functionalities.

\section{Experimental part}

Unless otherwise stated, all chemicals and solvents were of analytical grade and used without further purification.

\section{DLS measurements}

DLS measurements were performed at $22^{\circ} \mathrm{C}$ using a $173^{\circ}$ noninvasive backscatter method on a Malvern Instruments Zetasizer Nano ZS particle size analyser using $1 \mathrm{mg} \mathrm{mL}^{-1}$ solutions in Milli-Q water filtered through $450 \mathrm{~nm}$ PTFE filters unless stated otherwise. The data are averages of 3 measurements with each experiment repeated at least twice.

\section{Synthesis of PEG-Tyr block copolymer}

PEG2000-Tyr $6\left(M_{\mathrm{n}}=2959 \mathrm{~g} \mathrm{~mol}^{-1}\right)$ was prepared according to published literature procedures. ${ }^{5}$

\section{Spin labelling of PEG-Tyr}

PEG-Tyr copolymer was spin labelled using a procedure adapted from literature. ${ }^{7}$ PEG2000-Tyr 6 (1.33 mg) was dissolved in methanol $(100 \mu \mathrm{L})$ to give a $4.58 \mathrm{mM}$ solution. A $58.6 \mathrm{mM}$ amino-TEMPO solution in methanol $(50 \mu \mathrm{L})$ was added to the sample, followed by $3.5 \mathrm{M}$ formaldehyde solution in methanol $(8.4 \mu \mathrm{L})$. The solution was stirred at room temperature for $16 \mathrm{~h}$, and the solvent was removed under vacuum. The residue was redissolved in Milli-Q water $(333 \mu \mathrm{L})$ to give an end polymer concentration of $1.38 \mathrm{mM}$. The crude product was then dialysed in deionised water $(3 \mathrm{~L})$ to remove any unreacted nitroxide using a dialysis membrane ( $M_{\mathrm{w}}$ cut-off $\left.12-14 \mathrm{kDa}\right)$, with 2 water changes over 24 hours.

\section{EPR analysis of spin labelled samples}

$5 \mathrm{mg} \mathrm{mL}{ }^{-1}$ (1.72 mM) aqueous spin labelled PEG2000-Tyr 6 polymer solutions in Milli-Q water were placed into a quartz flat cell and X-band EPR spectra were recorded at room temperature on JEOL JES-X320 spectrometer. Typical EPR parameters: microwave power $=2 \mathrm{~mW}$, frequency $=9.128 \mathrm{GHz}$, sweep width $=15 \mathrm{mT}$, modulation frequency $=100 \mathrm{kHz}$, modulation width $=0.1 \mathrm{mT}$, sweep time $=240 \mathrm{~s}$, number of scans $=5$, time constant $=0.1 \mathrm{~s}$.

Gel samples were prepared by heating the micellar solutions within the flat cell in a water bath at $60{ }^{\circ} \mathrm{C}$. Gelation was confirmed by introducing a small metal (lead solder) ball to the top of the sample within the flat cell. Following gelation, the increased macroscopic viscosity of the sample prevented the ball sinking through the sample.

EPR spectra were simulated using EasySpin (chili function) ${ }^{9}$ to determine rotational correlation times of different spectral components.

\section{Low temperature Q-band EPR measurements}

$5 \mathrm{mg} \mathrm{mL}{ }^{-1}$ (1.72 mM) Aqueous spin labelled PEG2000-Tyr 6 polymer solutions in Milli-Q water were placed into a quartz EPR tube (inner diameter $1.1 \mathrm{~mm}$, Wilmad WG-221T-RB). The samples were then frozen in the EPR cavity. Spectra were recorded at a temperature of $88 \mathrm{~K}$. Typical EPR parameters: microwave power $=124 \mu \mathrm{W}$, frequency $=34.69 \mathrm{GHz}$, sweep width $=30 \mathrm{mT}$, modulation frequency $=100 \mathrm{kHz}$, modulation width $=0.1 \mathrm{mT}$, sweep time $=480 \mathrm{~s}$, number of scans $=10$, time constant $=0.3 \mathrm{~s}$.

EPR spectra were simulated using EasySpin (pepper function) ${ }^{9}$ to determine principal values of $g$ - and $A$-tensors.

\section{Monitoring gelation in situ by EPR}

$10 \mathrm{mg} \mathrm{mL}{ }^{-1}$ (3.44 mM) Aqueous spin labelled PEG2000-Tyr polymer solutions were heated to $60{ }^{\circ} \mathrm{C}$ by immersing the sealed quartz flat cell in a water bath at the required temperature. After heating for 30 seconds, the sample was removed, allowed to cool to room temperature for 5 minutes before reanalysing by EPR as described above. This process was repeated multiple times to monitor the change by EPR over 30 minutes heating.

\section{Photochemical degradation measurements}

$5 \mathrm{mg} \mathrm{mL} \mathrm{m}^{-1}(1.72 \mathrm{mM})$ aqueous samples of PEG2000-Tyr 6 $(280 \mu \mathrm{L})$ were prepared in $0.1 \mathrm{mM}$ acetate buffer at $\mathrm{pH} 5$. Samples were then split into two quartz cuvettes: one sample $(140 \mu \mathrm{L})$ was left in the dark as a control, the other sample was irradiated for 24 hours with a $100 \mathrm{~W}$ high pressure Hg lamp. Following irradiation, the samples were diluted to a volume of $1 \mathrm{~mL}$ with ethanol (140 $\mu \mathrm{L}$, to break up micelle/gel structures), $0.1 \mathrm{~mol} \mathrm{dm}^{-3}$ acetate buffer at pH $5(400 \mu \mathrm{L})$ and $0.4 \mathrm{~mol} \mathrm{dm}^{-3}$ borate buffer at $\mathrm{pH} 8.5(400 \mu \mathrm{L})$, to bring the end $\mathrm{pH}$ to 8.5 (for improved observation of singly deprotonated dityrosine fluorescence). ${ }^{15}$ Samples were analysed by fluorometry for characteristic tyrosine fluorescence: excitation scan 240$300 \mathrm{~nm}$ with $\lambda_{\mathrm{em}}=303 \mathrm{~nm}$, emission scan $270-500 \mathrm{~nm}$ with $\lambda_{\text {ex }}=276 \mathrm{~nm}$. Samples were also analysed for dityrosine 
fluorescence: excitation scan $240-400 \mathrm{~nm}$ with $\lambda_{\mathrm{em}}=405 \mathrm{~nm}$, emission scan $325-500 \mathrm{~nm}$ with $\lambda_{\mathrm{ex}}=320 \mathrm{~nm}$. The degradation of tyrosine fluorescence and increase in dityrosine fluorescence was determined relative to the dark control for every experiment.

Gel samples were prepared as described above, with heating in a water bath at $60{ }^{\circ} \mathrm{C}$ for one hour after splitting the PEG-Tyr sample into two cuvettes. Gelation was confirmed after this time by inverting cuvettes, and samples were analysed as described above.

Hydrophobic iron(III) acetylacetonate was added to some samples. $\mathrm{Fe}(\mathrm{acac})_{3}$ stock solution at a metal concentration of $50 \mathrm{ppm}$ was prepared in water. This stock solution $(0.56 \mu \mathrm{L})$ was added to $5 \mathrm{mg} \mathrm{mL}^{-1}(1.72 \mathrm{mM})$ samples of PEG-Tyr copolymer $(280 \mu \mathrm{L})$ in $0.1 \mathrm{M}$ pH 5 acetate buffer to give a metal concentration of $0.1 \mathrm{ppm}$. Photochemical experiments were then performed as described above.

\section{Conflicts of interest}

There are no conflicts to declare.

\section{Acknowledgements}

The authors acknowledge The Procter \& Gamble Company for support of this work.

\section{Notes and references}

1 K. Bhattacharyya, Acc. Chem. Res., 2003, 36, 95-101; A. V. Davis, R. M. Yeh and K. N. Raymond, Proc. Natl. Acad. Sci. U. S. A., 2002, 99, 4793-4796; N. J. Buurma, Annu. Rep. Prog. Chem., Sect. B: Org. Chem., 2012, 108, 316-333.

2 A. Galana and P. Ballester, Chem. Soc. Rev., 2016, 45, 1720-1737; P. Mal, B. Breiner, K. Rissanen and J. R. Nitschke, Science, 2009, 324, 1697-1699.
3 D. G. Whitten, Acc. Chem. Res., 1993, 26, 502-509; A. Dey and K. Biradha, Isr. J. Chem., 2019, 59, 220-232.

4 J. L. Santos and M. Herrera-Alonso, Macromolecules, 2014, 47, 137-145.

5 J. Huang, C. L. Hastings, G. P. Duffy, H. M. Kelly, J. Raeburn, D. J. Adams and A. Heise, Biomacromolecules, 2013, 14, 200-206.

6 G. H. Van Domeselaar, G. S. Kwon, L. C. Andrew and D. S. Wishart, Colloids Surf., B, 2003, 30, 323-334.

7 M. Lorenzi, C. Puppo, R. Lebrun, S. Lignon, V. Roubaud, M. Martinho, E. Mileo, P. Tordo, S. R. A. Marque, B. Gontero, B. Guigliarelli and V. Belle, Angew. Chem., Int. Ed., 2011, 50, 9108-9111.

8 E. Mileo, E. Etienne, M. Martinho, R. Lebrun, V. Roubaud, P. Tordo, B. Gontero, B. Guigliarelli, S. R. A. Marque and V. Belle, Bioconjugate Chem., 2013, 24, 1110-1117.

9 S. Stoll and A. Schweiger, J. Magn. Reson., 2006, 178, 42-55. 10 J. P. Klare, Biol. Chem., 2013, 394, 1281-1300.

11 C. Giulivi, N. J. Traaseth and K. J. A. Davies, Amino Acids, 2003, 25, 227-232.

12 D. I. Pattison, A. S. Rahmanto and M. J. Davies, Photochem. Photobiol. Sci., 2012, 11, 38-53.

13 W. A. Pryor, Annu. Rev. Physiol., 1986, 48, 657-667; R. B. Mikkelsen and P. Wardman, Oncogene, 2003, 22, 5734-5754.

14 G. Ruppert, R. Bauer and G. Heisler, J. Photochem. Photobiol., A, 1993, 73, 75-78; P. Chábera, Y. Liu, O. Prakash, E. Thyrhaug, A. E. Nahhas, A. Honarfar, S. Essén, L. A. Fredin, T. C. Harlang, K. S. Kjær, K. Handrup, F. Ericson, H. Tatsuno, K. Morgan, J. Schnadt, L. Häggström, T. Ericsson, A. Sobkowiak, S. Lidin, P. Huang, S. Styring, J. Uhlig, J. Bendix, R. Lomoth, V. Sundström, P. Persson and K. Wärnmark, Nature, 2017, 543, 695-699.

15 M. Correia, M. T. Neves-Petersen, P. B. Jeppesen, S. Gregersen and S. B. Petersen, PLoS One, 2012, 7, e50733. 